\section{NRG1 meets resistance}

\section{By Lev Osherovich, Senior Writer}

Researchers at Roche's Genentech Inc. unit have shown that the receptor tyrosine kinase ligand neuregulin 1 can drive resistance to chemotherapy in non-small cell lung cancers. Although the precise mechanism is being worked out, countering resistance by hitting neuregulin 1 or its receptors in conjunction with conventional chemotherapy could prevent chemo resistance. ${ }^{1}$

Neuregulin 1 (NRG1) is an autocrine signaling factor that normally promotes survival and differentiation in developing tissues. The protein acts on several receptors, including epidermal growth factor receptor 3 (EGFR3; HER3; ErbB3) and HER4 (EGFR4; ErbB4), and recently has turned up as a marker of EGFR inhibitor resistance in several tumor types. ${ }^{2}$

Now, a team led by Genentech principal scientist Erica Jackson has found that NRG1 signaling through HER3 and HER4 is more than just a marker and actually drives chemotherapeutic resistance in non-small cell lung cancer (NSCLC).

Although the specific mechanism of how the pathway protects tumor cells remains unclear, the company has shown that NRG1-blocking mAbs can prevent the emergence of chemo-resistant tumor cells.

Jackson's team found that NRG1-HER3/HER4 signaling was higher in tumor cells that had survived a course of chemotherapy than in treatment-naïve tumors.

The group suspected that these cells were present in the original tumor and proliferated after chemotherapy, which killed off the cells lacking this pathway but left NRG1-overexpressing cells intact.

Indeed, in a variety of mouse models of NSCLC, "only a subset of initial cells had active NRG1 signaling, but this correlated with resistance" to chemotherapy, said Jackson.

The team found that anti-NRG1 mAbs did not greatly decrease tumor growth or increase the survival of mice when given as a single agent compared with control mAbs. However, anti-NRG1 mAbs significantly decreased tumor size and increased progression-free survival when combined with chemotherapy compared with control antibodies plus chemotherapy.

Thus, Jackson suspected that blocking NRG1 signaling would prevent the emergence of chemotherapy-resistant cells after treatment.
Indeed, blocking either Her3 or Her4 signaling with small hairpin RNA knockdown or mAbs decreased tumor survival after chemotherapy compared with no blocking.

On the whole, the findings point to NRG1-HER3/HER4 signaling as a survival mechanism for a fraction of cells in NSCLCs (see Figure 1, "Neuregulin signaling in chemotherapy-resistant lung cancer"). According to this model, chemotherapy preferentially kills off cells that lack the pathway, leading the NRG1-HER3/HER4 minority to become the majority.

Results were published in Science Translational Medicine.

\section{High NRG1}

Jackson said that although mAbs against NRG1 and its receptors could in principle be used as single-agent therapeutics in tumors that already have high activity of the NRG1-HER3/HER4 pathway, "a broader use would be in combination with chemotherapy."

She added, "I don't know if there's a precedent for using an antibody to prevent resistance."

Bill Gullick, professor of cancer biology at University of Kent, said that HER3 has previously been considered a target for chemotherapeutic-resistant tumors and that Jackson's study fleshes out the pathway and points to new targets.

"Antibodies to HER3 are already in several clinical trials as anticancer drugs," said Gullick. "HER4, as far as I am aware, has yet to be tried, but there are several antibodies being lined up for this, and there are publications on their preclinical effects."

In 2011, Gullick's team reported that antibodies against HER3 blocked NRG1 binding and enhanced the efficacy of Herceptin trastuzumab, a mAb that inhibits HER2 (EGFR2; ErbB2; neu), a related receptor. ${ }^{3}$

Herceptin is marketed by Roche to treat breast cancer.

Jackson's next step is to look at NSCLC samples before and after chemotherapy to spot the emergence of NRG1-driven resistance. She said that in NSCLC, chemotherapy resistance usually develops just prior to end-stage disease, at which point the tumors are considered inoperable. Thus, obtaining post-chemotherapy samples could be tricky because chemotherapy-resistant tumors are not typically biopsied.

The biggest question now is why NRG1-HER3/HER4 signaling promotes survival. Jackson said her team is investigating the downstream consequences of effects of this pathway to see whether known prosurvival players such as B cell lymphoma 2 (BCL-2; BCL2) or phosphoinositide 3-kinase (PI3K) become activated.

"HER3 couples particularly well to the PI3K pathway, which is involved in promoting cell survival by avoiding apoptosis," Gullick noted.

Another possibility is that cells with active NRG1-HER3/HER4 signaling grow more slowly than other tumor cells and are thus protected from chemotherapeutics that target proliferating cells. 


\section{ANALYSIS}

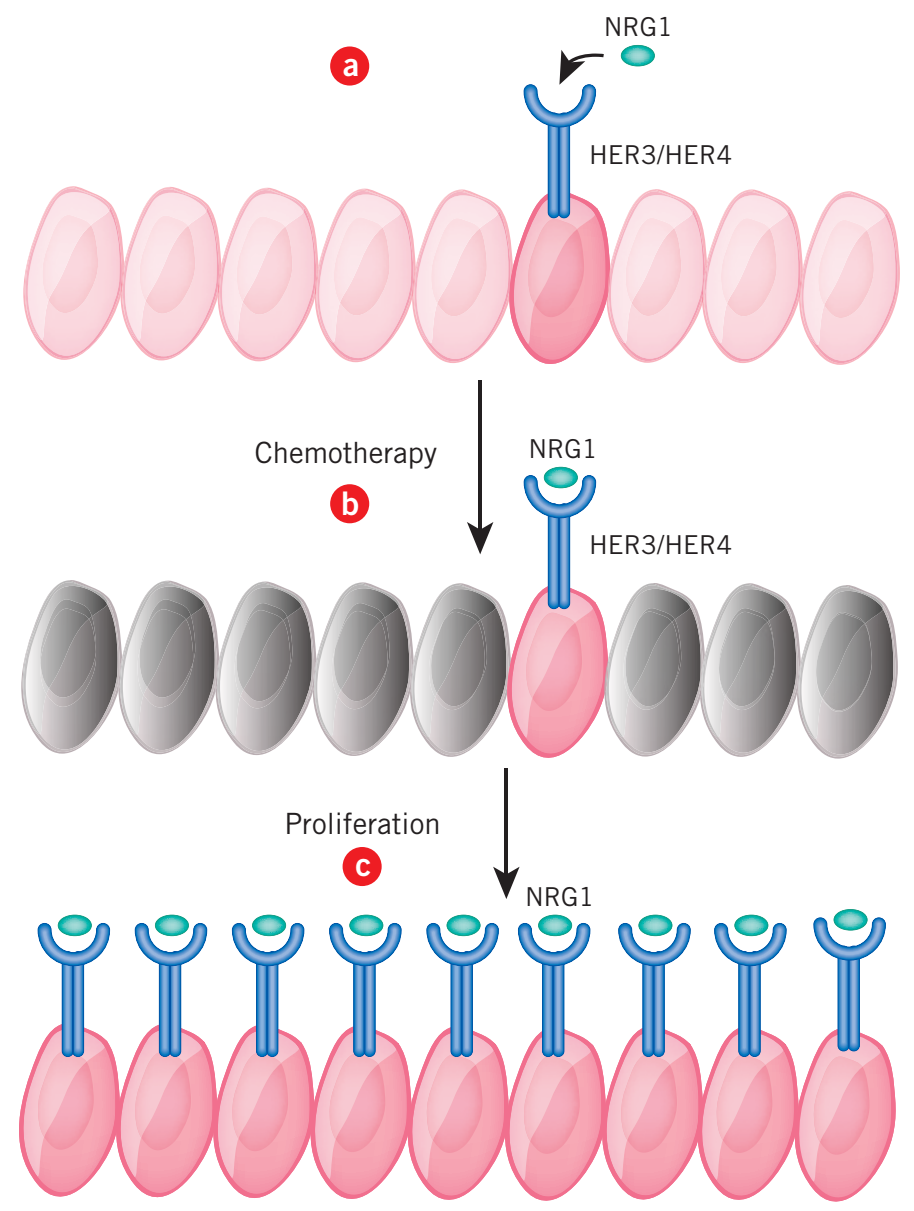

Regardless of the pathway's mechanism of action, another question is how best to target NRG1, HER3 or HER4 to improve the efficacy of chemotherapy.

Gullick thinks that hitting NRG1 itself will be challenging because it is one of four closely related EGFR ligands that may have overlapping effects on tumor survival.

"I would be concerned as to how much effect an inhibitor to only one of these would have," said Gullick. Instead, Gullick advocates hitting the receptors, not the ligand.

"In my opinion antibodies to HER3 and HER4 are more likely to be effective in combination with other targeted reagents to HER2 and EGFR and possibly in combination with conventional cytotoxic drugs," said Gullick.
Figure 1. Neuregulin signaling in chemotherapy-resistant lung cancer. Hegde et al. have uncovered a mechanism for chemotherapy resistance in non-small cell lung cancer (NSCLC).

In NSCLC tumors, a small number of cancer cells have high levels of neuregulin 1 (NRG1), an autocrine signaling protein that activates epidermal growth factor receptor 3 (EGFR3; HER3; ErbB3) and HER4 (EGFR4; ErbB4) [a]. Those cells are protected from the toxic effects of a variety of chemotherapeutic agents [b] and go on to proliferate after chemotherapy to reconstitute the tumor [c].

Roche's Genentech Inc. unit has discovery stage antibodies against NRG1 that prevent survival of NRG1-expressing cells during chemotherapy.

Along these lines, Genentech's RG7597 (MEHD7945A), a mAb that targets HER3 and the closely related EGFR, is in Phase II trials for a variety of solid tumors. It is unclear whether that $\mathrm{mAb}$ would prevent the emergence of chemotherapeutic resistance.

In the Science Translational Medicine study, Jackson's team improved chemotherapeutic efficacy using mAbs against all three proteins as well as with a HER4 extracellular domain that traps NRG1 and blocks its activity. Small molecule inhibitors of HER3 or HER4 kinase activity could be another option.

Genentech has filed for patents covering the use of anti-NRG1 mAbs to treat cancer. Jackson said the company has not yet chosen a lead candidate for the program, which she described as being at the discovery stage.

Osherovich, L. SciBX 6(7); doi:10.1038/scibx.2013.155

Published online Feb. 21, 2013

\section{REFERENCES}

1. Hegde, G.V. et al. Sci. Transl. Med.; published online Feb. 6, 2013; doi:10.1126/scitransImed.3004438

Contact: Erica L. Jackson, Genentech Inc., South San Francisco, Calif. e-mail: ericaj@gene.com

2. Dhomen, N.S. et al. Crit. Rev. Oncog. 17, 31-50 (2012)

3. Blackburn, E. et al. Breast Cancer Res. Treat. 134, 53-59 (2012)

\section{COMPANIES AND INSTITUTIONS MENTIONED}

Genentech Inc., South San Francisco, Calif.

Roche (SIX:ROG; OTCQX:RHHBY), Basel, Switzerland University of Kent, Canterbury, U.K. 